\title{
Isolated Absent Ductus Venosus with Intrahepatic Shunt: Case Report and Review of Literature
}

\author{
Rajeev Choudhary $\cdot$ Sandeep Bajaj Choudhary
}

Received: 1 September 2014 / Accepted: 26 September 2014/Published online: 9 October 2014

(C) Society of Fetal Medicine 2014

\begin{abstract}
A 28-year-old primigravida with spontaneous conception and no complicating medical illness presented to the authors for an early morphology scan. Her scan was unremarkable with normal nuchal translucency for crown rump length, no stigmata for chromosomal abnormalities and no major detectable structural abnormality. A repeated search for ductus venosus revealed non visualization of the ductus with intrahepatic drainage of the umbilical vein. The karyotype was normal. Fetal echocardiogram, anomaly and serial growth scans were also within normal limits. She developed mild hydramnios in the late third trimester and delivered a normal healthy female child at 37 weeks. Postnatal echocardiogram showed a very small muscular ventricular septal defect. Mild pulmonary stenosis has been diagnosed at the age of 6 months. Ductus venosus is the key regulator of oxygenated blood in fetal life. Absent ductus venosus is associated with multiple structural, chromosomal, cardiac abnormalities partial or complete absence of portal venous system, hydrops and fetal death. In the absence of ductus venosus the umbilical vein may drain normally into the liver (intrahepatic shunt) or may drain in an aberrant location into the systemic circulation (e.g., iliac vein, inferior vena cava, right heart and coronary sinus) creating an extrahepatic shunt. In all the cases of absent ductus venosus, the prognosis depends on the presence or absence of associated abnormalities. In cases of extrahepatic aberrant drainage of the umbilical vein, the prognosis depends upon the presence or absence of portal venous system, abnormal shunt site and shunt diameter. In
\end{abstract}

R. Choudhary $(\bowtie) \cdot S$. B. Choudhary

Centre for Fetal Medicine, Dr Rajeev's Ultrasound Lab, B-15,

Shankar Garden, Vikas Puri, New Delhi 110018, India

e-mail: dr_rajeevchoudhary@yahoo.com general, intrahepatic variant has a much better prognosis in the absence of other abnormalities.

Keywords Absent ductus venosus - Intrahepatic shunt . Extrahepatic shunt - Congenital absence of portal venous system - Aberrant drainage of umbilical vein .

Turner syndrome

\section{Introduction}

By the seventh gestational week, three pairs of major veins connect to the primitive heart $[1,2]-$ the vitelline veins draining blood from the yolk sac and primitive midgut; the cardinal veins, collecting blood from the embryo; the umbilical veins, carrying the oxygenated blood from the placenta to the embryo. The right umbilical vein degenerates leaving the entire placental venous return to the left umbilical vein which connects to the left portal vein. Later a direct connection is established between the left portal vein (PV) and systemic circulation-the ductus venosus (DV). Embryologically, the failure of 'critical anastamosis' between the umbilical and portal venous system and hepatic-systemic venous system results in absent ductus venosus (ADV).

The DV is a funnel shaped vessel connecting the intraabdominal umbilical vein (UV) and the portal sinus with the inferior vena cava (IVC). In fetal life, the UV returns oxygenated blood from the placenta to the portal sinus and DV. The narrowed portal sinus end of the DV regulates blood flow and under normal conditions, allows $20-30 \%$ of the oxygenated umbilical venous return to bypass the liver and portal venous circulation and enter the proximal IVC [2], thus allowing the oxygenated blood to reach the coronary 

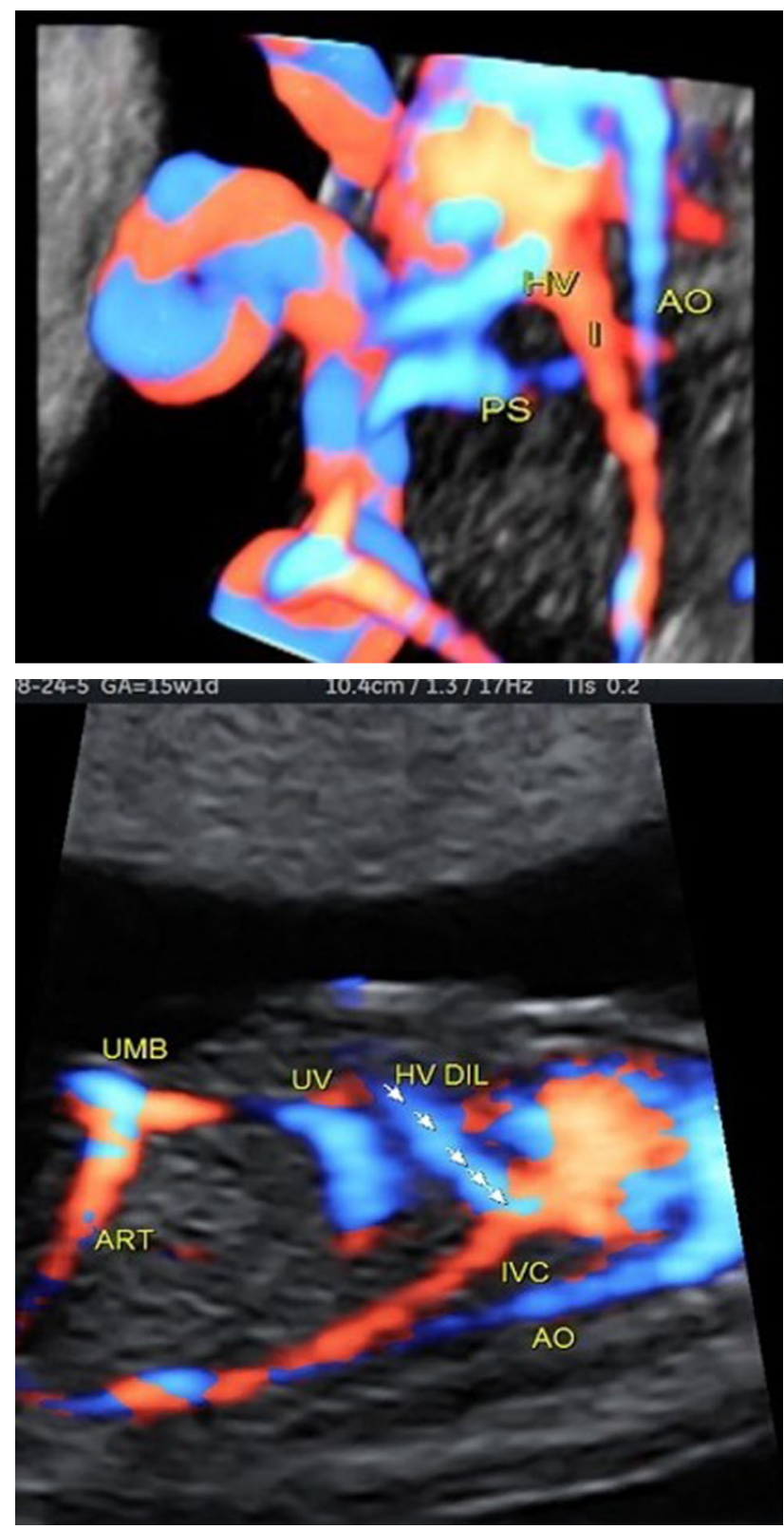

Fig. 1-2 Power Doppler and 3D power Doppler showing absence of normal ductus venosus at its usual location

and cephalic circulations rapidly. The balance of umbilical venous flow enters the portal circulation and streams predominantly to the left lobe of the liver. The structure of the DV controls the shunt from the umbilical vein to the IVC and produces a high velocity jet directed to the foramen ovale [1-3]. With hypoxia or hypovolemia the DV may dilate to increase the percentage of oxygenated umbilical venous flow to the IVC and hence to the cephalic and coronary circulations. Postnatally the DV collapses and undergoes fibrotic transformation resulting in ligamentum venosum.

Ductus venosus is a very important regulator of oxygenated blood in fetal life. Current improvement in 2D and

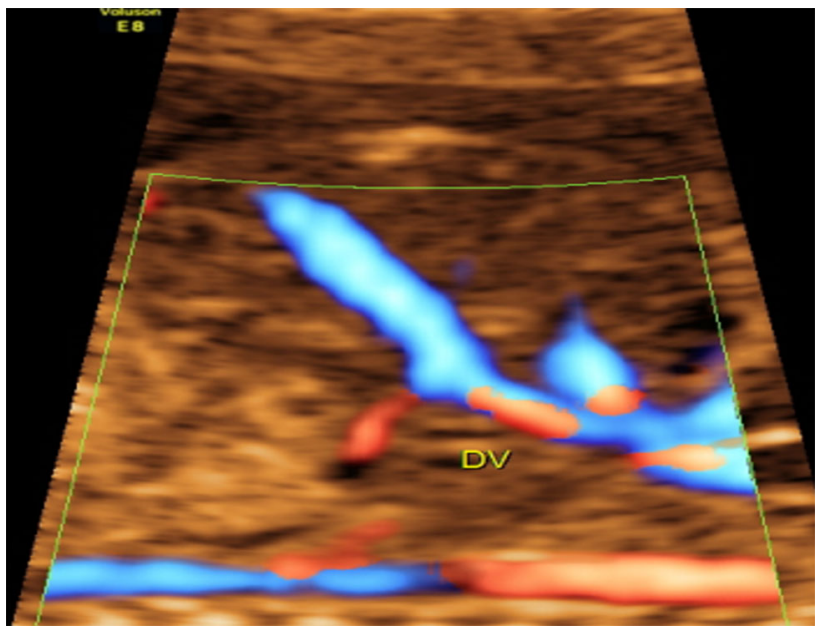

Fig. 3 Shows normal ductus venosus for comparison

3D ultrasound techniques and incorporation of DV Doppler evaluation in late first trimester allow us to detect many umbilical-portal venous abnormalities including absent DV which is associated with many malformations and cytogenetic abnormalities [4]. Ductus venosus flows are routinely used for refining diagnosis of cardiac, extra cardiac and chromosomal anomalies and first trimester screening for Down's syndrome. Ductus venosus pulsatility index (PI) can be used as a continuous variable with nuchal translucency (NT) to increase specificity in the identification of congenital heart diseases [5]. The authors present here a case of ADV with intrahepatic drainage of UV (Intrahepatic shunt).

\section{Report of Case}

A 28-year-old gravida one para 0 with spontaneous conception and no pre-existing medical condition presented to the authors for an early morphology scan at 14 weeks gestation. A repeated search for DV including color and power Doppler and high definition flows resulted in the non visualization of the DV (Figs. 1-2, 3 shows a normal DV for comparison). The UV course was normal draining into the liver and portal vein which appeared mildly dilated. Her NT measurement was $2.0 \mathrm{~mm}$ and a detailed evaluation did not reveal any morphological, apparent cardiac abnormality or stigmata of chromosomal abnormalities. She was offered a karyotype by amniocentesis which did not show any abnormality. Her serial ultrasound scans including the anomaly scan, fetal echocardiography, growth scans and Doppler flows in other vessels during the pregnancy were within normal limits. She developed mild hydramnios in the late third trimester scans and delivered a normal healthy $2,900 \mathrm{~g}$ female child at 37 weeks by caesarean section for loss of fetal movements. 
The newborn had an uneventful immediate postnatal period but showed a very small muscular ventricular septal defect (VSD) on echocardiography. She was later diagnosed with mild pulmonary stenosis at 6 months of age.

\section{Discussion}

Two abnormal different patterns of umbilical venous drainage are seen in cases of ADV [6]. The UV may connect directly to the portal vein without giving rise to the DV (intrahepatic shunt); UV may bypass the liver and may connect to the subdiaphragmatic IVC, renal veins or iliac veins; UV may directly connect to the right atrium intrahepatic IVC, coronary sinus or even left atrium (extrahepatic shunts). Absent ductus venosus with intrahepatic drainage has rarely been reported in literature [6-8]. Absent ductus venosus is an uncommon anomaly with reported incidence varying from 1 in 2,532 in a very large study of 65,840 pregnancies at 11-14 weeks [9], to 6/1,000 [3] in high risk cases. Absent ductus venosus is associated with morphological abnormalities in approximately $65 \%$ cases and cytogenetic abnormalities in $17 \%$ cases [4, 6].

The study by Staboulidou et al. [9] also showed a strong association of ADV with aneuploidies and a marked increase in the incidence of abnormal outcomes when ADV was associated with increased Nuchal translucency measurements (NTL) above 95th centile for crown rump length (CRL) $(66.7 \%$ incidence of aneuploidy, mostly Turner syndrome) as compared to cases when ADV was an isolated finding and associated with normal NTL measurements (81.8\% normal healthy live births).

Systemic abnormalities associated with ADV include [4, $6,10]$ cardiac (atrial and ventricular septal defects, tricuspid atresia, double outlet right ventricle pulmonary atresia and transposition of great arteries) gastrointestinal (duodenal atresia, tracheoesophageal fistula) genitourinary system (hydronephrosis, ectopic kidney) and musculoskeletal abnormalities. One of the most critical associations of ADV is the complete or partial agenesis of the portal venous system [11] usually in cases where the UV bypasses the liver resulting in severe postnatal complications like pulmonary edema, focal nodular hypoplasia and hepatic tumors. The presence of associated chromosomal and structural abnormalities determines the prognosis in both intrahepatic and extrahepatic drainage of the UV. In cases of extrahepatic drainage of the UV, the prognosis, additionally, depends on the extent of development of the portal venous system and its resultant complications, the site of drainage of the UV (shunt site) [6], and the shunt diameter [12] in relation to the umbilical vein diameter. Closer the UV drains to the right heart bypassing the liver, presumably, more the preload and more the likelihood of intrauterine heart failure. The farther the UV drains from the right heart and narrower the shunt diameter, lesser the preload, lower the likelihood of intrauterine heart failure. Cases with relatively smaller shunt diameters, compared to the UV diameter show better development of the portal venous system and hence better outcome [6, 11, 12]. In general, extrahepatic umbilical venous drainage results in worst fetal outcomes compared to intrahepatic drainage of the UV $[6,8,11,12]$.

Gembruch et al. [7] reported two cases of intrahepatic drainage of the umbilical vein in 1998 with ADV in second and third trimesters without the development of hydrops or compromising fetal hemodynamics. They suggested that absence of the DV may be compatible with normal fetal development without relevant disturbance of circulation and oxygenation. Berg et al. [6] also concluded that ADV without liver bypass seems to have better prognosis in the absence of other malformations. In cases of intrahepatic shunts, as in the present case, the presence of a normal portal venous system and an intervening high resistance bed between the UV and right heart (hepatic venous sinusoids and hepatic veins) probably prevents hepatic complications and increased preload [3].

The commonest cause of nonvisualization of DV is technical, resulting from unfriendly fetal positions, maternal body habitus and surgical scars. Repeated examinations are a must to exclude technical cause of DV non visualization. Modern ultrasound equipment allow excellent multiplanar visualization of the umbilical-portal venous anatomy in 2D, Doppler and 3D power Doppler modes for enhanced diagnostic confidence [10]. In cases of confirmed ADV the normalcy of portal venous system, site of UV drainage (intrahepatic or extrahepatic) and diameter of the abnormal shunt in relation to the caliber of the UV should be ascertained. Karyotype, a detailed morphological and cardiac assessment in expert hands is also important. The patient should be counseled regarding the complications and the risk of intrauterine fetal death, hydrops, cardiac failure and expected postnatal course. Delivery should preferably be conducted in a tertiary center with facilities to manage complications including surgical or device closure of abnormal shunts [3]. Recurrence risk of ADV is not known but probably depends on the associated chromosomal and structural abnormalities.

Conflict of interest None. 


\section{References}

1. Shen O, Tadmor OP, Yagel S. Prenatal diagnosis of persistent right umbilical vein. Ultrasound Obstet Gynecol. 1996;8:31-3.

2. Kiserud T, Rasmussen S, Skulstad S. Blood flow and the degree of shunting through the ductus venosus in the human fetus. Am J Obstet Gynecol. 2000;182:147-53.

3. Acherman RJ, Evans WN, Galindo A, et al. Diagnosis of absent ductus venosus in a population referred for fetal echocardiography: association with a persistent portosystemic shunt requiring postnatal device occlusion. J Ultrasound Med. 2007;26:1077-82.

4. Volpe P, Marasini M, Caruso G, et al. Prenatal diagnosis of ductus venosus agenesis and its association with cytogenetic/ congenital anomalies. Prenat Diagn. 2002;22:995-1000.

5. Timmerman E, Clur SA, Pajkrt E, et al. First trimester measurement of the ductus venosus pulsatility index and the prediction of congenital heart defects. Ultrasound Obstet Gynecol. 2010;36:668-75.

6. Berg C, Kamil D, Giepel A, et al. Absence of ductus venosusImportance of umbilical venous drainage site. Ultrasound Obstet Gynecol. 2006;28:275-81.
7. Gembruch U, Baschat AA, Caliebe A, et al. Prenatal diagnosis of ductus venosus agenesis: a report of two cases and review of the literature. Ultrasound Obstet Gynecol. 1998;11:185-9.

8. Contraatti G, Banzi C, Ghi T, et al. Absence of the ductus venosus: report of 10 new cases and review of literature. Ultrasound Obstet Gynecol. 2001;18:605-9.

9. Staboulidou I, Pereira S, de Jesus Cruz J, et al. Prevalence and outcome of absence of ductus venosus at $11(+0)$ to $13(+6)$ weeks. Fetal Diagn Ther. 2011;30:35-40.

10. Thubert T, Levaillant JM, Stos B, et al. Agenesis of ductus venosus: three-dimensional power doppler reconstruction. Ultrasound Obstet Gynecol. 2012;39:118-20.

11. Achiron R, Gindes L, Kevilevitchz Z, et al. Prenatal diagnosis of congenital agenesis of the fetal portal venous system. Ultrasound Obstet Gynecol. 2009;34:643-52.

12. Shen O, Valsky DV, Messing B, et al. Shunt diameter in agenesis of the ductus venosus with extrahepatic portosystemic shunt impacts on prognosis. Ultrasound Obstet Gynecol. 2011;37:18490. 